\title{
Characterization of Atmospheric Pressure Plasmas by a Gerdien Condenser*)
}

\author{
Ma Camille C. LACDAN and Motoi WADA \\ Graduate School of Science and Engineering, Doshisha University, Kyotanabe, Kyoto 610-0321, Japan
}

(Received 3 December 2015 / Accepted 26 January 2016)

\begin{abstract}
A compact Gerdien condenser is being developed as a new characterization tool for laboratory-produced atmospheric pressure plasma. The Gerdien condenser is capable of determining the ion density and mobility, thus ion species composition of the plasma. The device has a $1.0 \mathrm{~cm}$ diameter, $6.0 \mathrm{~cm}$ long cylindrical current collector electrode mounted on a bias electrode of $1.7 \mathrm{~cm}$ inner diameter. The flow rate of the fan attached at the end of the condenser is set from 1.9 to $7.5 \times 10^{2} \mathrm{~cm}^{3} \mathrm{~s}^{-1}$. Some of the values of ion mobility calculated from the $I-V$ characteristics obtained for atmospheric plasmas produced by RF excited atmospheric pressure plasma pen are in the range of $\mathrm{O}^{+}, \mathrm{O}^{-}, \mathrm{O}_{2}^{-}, \mathrm{O}_{2}{ }^{+}, \mathrm{Ar}^{+}, \mathrm{N}^{+}$and $\mathrm{N}_{2}{ }^{+}$. The total positive ion densities at $2.0 \mathrm{~cm}$ away from the plasma pen are determined to be in the range of $10^{6} \mathrm{~cm}^{-3}$.
\end{abstract}

(C) 2016 The Japan Society of Plasma Science and Nuclear Fusion Research

Keywords: Gerdien condenser, plasma diagnostics, atmospheric pressure plasma, ion mobility, ion density

DOI: $10.1585 /$ pfr. 11.2401015

\section{Introduction}

Plasma diagnostics plays an essential role in understanding the relationship of plasma parameters to the effectiveness in utilizing plasmas in fields such as biomedicine, nanotechnology and surface processing. There have been a lot of studies on plasma diagnostics for low-pressure plasmas, but a little information is available for atmospheric plasma case. For example, there are several reports on charged particle exposure to inactivate microorganisms; however, the exact role of ions is not yet fully understood [1]. It is of great interest to determine the plasma parameters in order to explain the mechanism behind the effectiveness of plasma processing under atmospheric pressure.

One of the common methods of plasma diagnostics is the Langmuir probe technique. The simplicity of its construction with ease adaptive to laboratory application and more importantly, its capability for local measurements of plasma parameters have been the primary reasons for the Langmuir probes to be widely used today. However, as it is made small for determining local plasma parameters, the Langmuir probe does not yield enough signal amplitude for dilute atmospheric pressure plasma. Langmuir probe technique requires that the ion production rate of plasma discharge to be sufficient so that the current intensity overcomes background noise. There have been attempts [2-4] to use Langmuir probes for atmospheric pressure plasmas, but the theory for atmospheric pressure plasma largely differs from the one for reduced pressure plasma.

One diagnostic method has been widely used in the stratosphere region for determining ions employing a Ger-

author'se-mail:euo1309@mail4.doshisha.ac.jp

*) This article is based on the presentation at the 25 th International Toki Conference (ITC25).

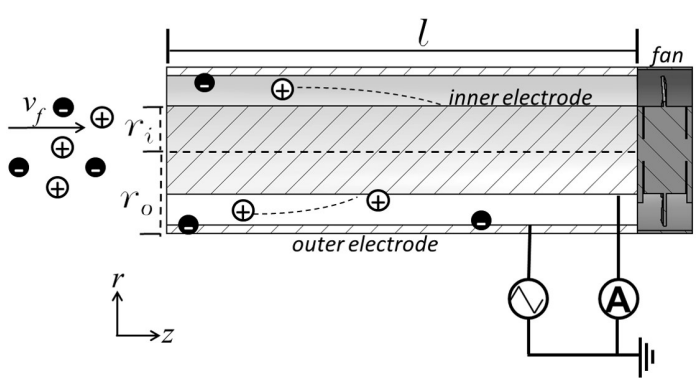

Fig. 1 Operation principle of Gerdien condenser.

dien condenser [5-9]. A Gerdien condenser can determine ion density with the mobility, thus ion species composition of plasma. Since the condenser actively takes in ions from the atmosphere, the method can be applicable even for characterizing dilute atmospheric-pressure plasmas where the input power for plasma production is less than $100 \mathrm{~W}$.

Figure 1 shows the principle of operation of a Gerdien condenser. The condenser takes in ions through a conduit with a biased cylindrical hollow outer electrode and an inner electrode which detects the current due to ion flow. The conduit is sufficiently ventilated since the mobility to be computed is determined from a signal proportional to the air flow rate [7].

If air containing positive ions of single species enters the condenser at a constant velocity $v_{\mathrm{f}}$ due to airflow driven by a fan, the current as a function of the applied voltage $V_{\text {in }}$ should be as shown in Fig. 2. The current-voltage $(I-V)$ characteristic is linear until the voltage $V_{\mathrm{s}}$ reaches the value at which all of the positive ions are collected by the inner electrode. The slope of the linear region determines the ion mobility $\mu_{k}$, hence the ion species present in the plasma [8]. 


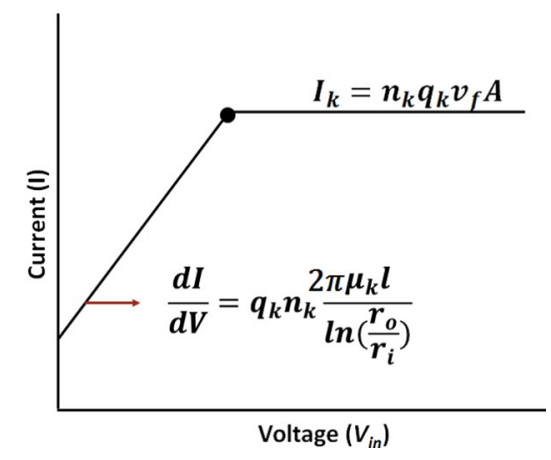

Fig. 2 Ideal current-voltage characteristics of a Gerdien condenser for gas containing single mobility.

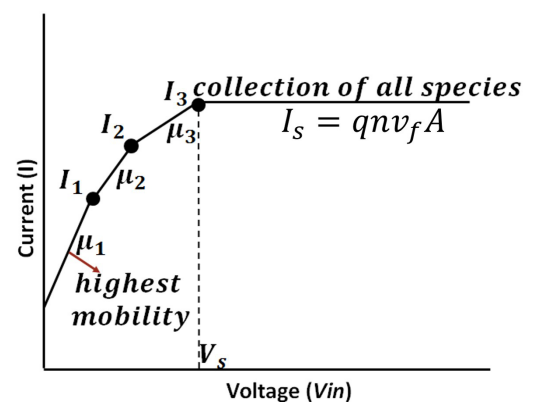

Fig. 3 Ideal current-voltage characteristics of a Gerdien condenser for multiple-constituent gas.

In practice, ambient air is a multiple-ion component gas. Each ion species has its own mobility, and the total current will be due to a sum of each ion species. A theoretical curve consisting of straight line segments for three mobilities is illustrated in Fig. 3. The current-voltage relationship will remain linear until all ions having the highest mobility are collected. At this point, the slope of the curve becomes zero due to saturation current of the highest mobility ion. As the voltage is increased, bending points or the knees are observed in the curve. All ions of lower mobilities are collected so that the slopes become smaller with increase of applied potential.

In this study, the applicability of Gerdien condenser as a diagnostic tool for laboratory-produced atmospheric pressure plasma is investigated. Since the ventilation of the Gerdien condenser plays an important role in the measured $I-V$ characteristics, the dependence of ion mobility and their corresponding density as the air flow rate is varied is also investigated.

\section{Methodology}

Figure 4 shows the schematic diagrams of the atmospheric pressure plasma (APP) devices. The APP successfully ignites and sustains discharge with a $13.56 \mathrm{MHz}$ frequency power source at a forward input power of $30 \mathrm{~W}$. The impedance of the setups are tuned such that the reflected power is as low as $2 \mathrm{~W}$. Flow rate of Ar maintained
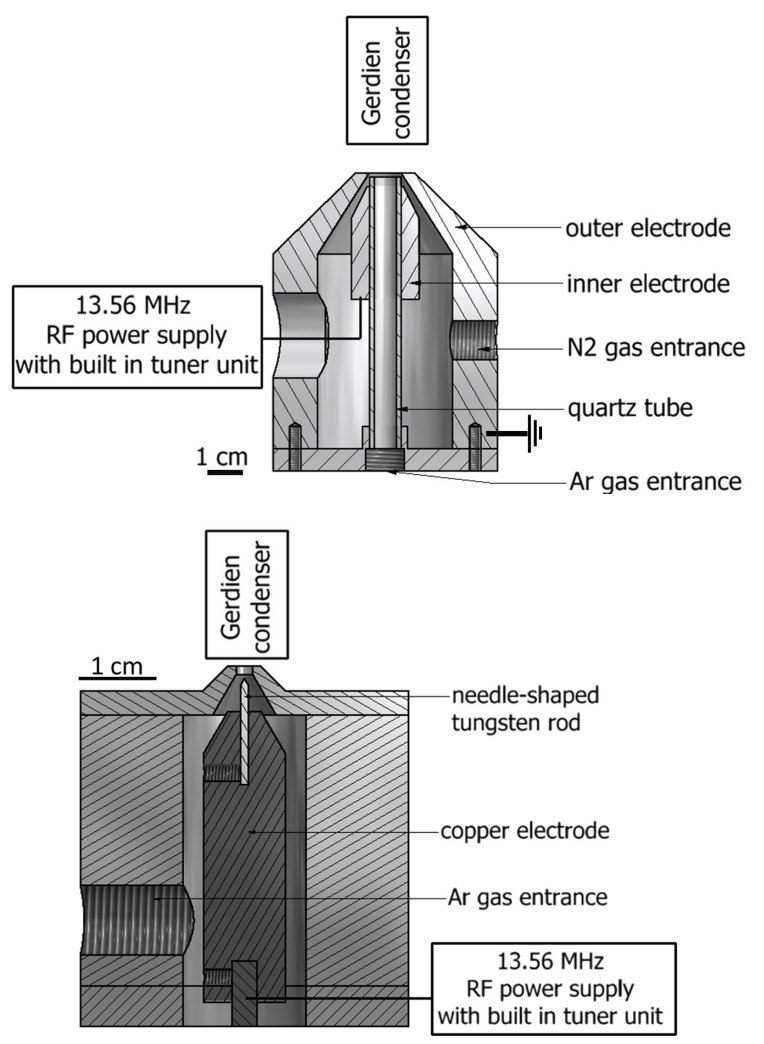

Fig. 4 Experimental setup of the atmospheric pressure plasma capacitively coupled configuration [top] and APP needleshaped electrode device [bottom].

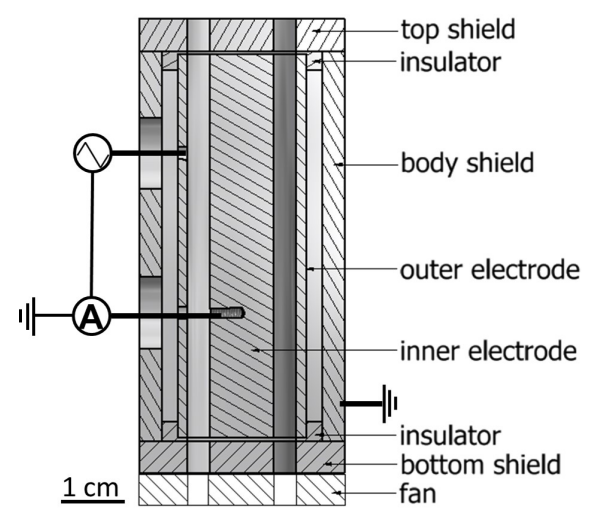

Fig. 5 Schematic diagram of the miniaturized Gerdien Condenser.

at 3 liters per minute $(\mathrm{L} / \mathrm{min})$ is supplied as the main gas to produce the plasma discharge. An additional $\mathrm{N}_{2}$ of $3 \mathrm{~L} / \mathrm{min}$ flow is used as the swirl gas to aid the plume of the APP out of the nozzle for the capacitively coupled configuration. The needle-shaped electrode device produces denser plasma compared to the capacitively coupled configuration at same input RF parameters.

A $6.0 \mathrm{~cm}$ long Gerdien condenser with $0.85 \mathrm{~cm}$ and $0.5 \mathrm{~cm}$ outer and inner cylinder radius, respectively is designed and fabricated as shown in Fig. 5. The entire condenser is mounted in an electrically grounded aluminum 
made rectangular shield box. A $2.5 \mathrm{~cm}$ by $2.5 \mathrm{~cm}$ fan is attached to the end to ventilate the tube and drag in ions into the collecting electrode. The Gerdien condenser is positioned apart from the plasma exit nozzle with pen-to-inlet distance of $2.0 \mathrm{~cm}$. A voltage sweep from 0 to $15 \mathrm{~V}$ is applied to the outer electrode to collect positive ions while the current is measured at the inner electrode. $I-V$ characteristics are recorded for varying fan flow rates of 1.9, $3.8,5.6$ and $7.5 \times 10^{2} \mathrm{~cm}^{3} \mathrm{~s}^{-1}$. The ion mobilities and total ion densities are calculated from the obtained $I-V$ characteristics. A negative voltage sweep is also applied to the collecting electrode to test the capability of Gerdien condenser in measuring negative ion species.

\section{Results}

\subsection{Current offset}

Figure 6 shows the $I-V$ characteristics of APP from Gerdien condenser for different fan flow rates. An offset at $V_{\text {in }}=0$ is observed for all the $I-V$ characteristics obtained. This offset can be caused by simultaneous collection of both positive and negative charged particles present from air inside the conduit due to either larger diffusion of positive ions or positive space potential in the conduit. The total current $I_{\mathrm{T}}$ collected at this case taking into the account of the contribution of both positive and negative ions present in ambient air which is supposed proportional to total current flowing into the condenser times some factor determined by diffusion or space charge $K_{k}$ :

$$
I_{\mathrm{T}}=\sum_{k} K_{k}\left(q_{k}{ }^{+} n_{k}{ }^{+}+q_{k}{ }^{-} n_{k}{ }^{-}\right) v_{\mathrm{f}} A
$$

Where, $q_{k}$ is the charge of ion $k, n_{k}$ is its corresponding density, $v_{\mathrm{f}}$ is the ion velocity due to airflow driven by a fan and $A$ is the area of Gerdien condenser given by $\pi\left(r_{\mathrm{o}}^{2}-r_{\mathrm{i}}^{2}\right)$.

\subsection{Ion density and ion mobility spectrum}

Table 1 shows the total ion densities, $n$, calculated from the saturation point depicted in Fig. 3 namely,

$$
n=I_{\mathrm{s}} /\left(q v_{\mathrm{f}} A\right) \text {. }
$$

Where, $I_{\mathrm{s}}$ is the saturation current obtained at $V=V_{\mathrm{s}}$ when all of the positive ions are collected by the inner electrode.

The increase in fan flow rate affects the air flow field surrounding the plasma. This results in enhanced ionic recombination among species of the plasma in the ambient air, hence a decrease in the detected total ion density is observed. However, the detected density did not decrease in proportion to the fan flow rate. The smaller input gas flow rate of the needle-shaped electrode device should create an air flow field of faster recombination at the region between the Gerdien condenser and the APP devices.

The denser plasma produced by the needle-shaped electrode device yielded higher ion density ranging from 6 to $16 \times 10^{6} \mathrm{~cm}^{-3}$ compared to the capacitively coupled configuration with ion density from 2 to $4 \times 10^{6} \mathrm{~cm}^{-3}$.
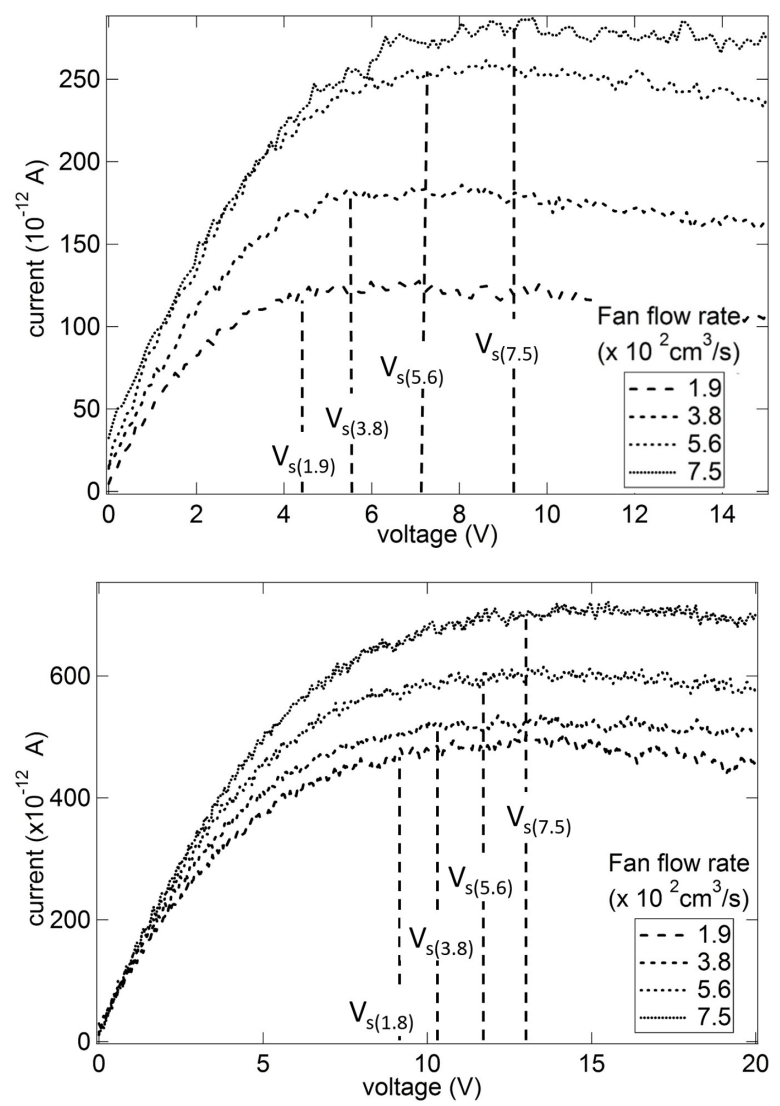

Fig. 6 Current-voltage characteristics produced by Gerdien condenser for the measured plasmas of capacitively coupled configuration [top] and needle-shaped electrode device [bottom] at varying fan flow rates. The dash lines mark the start of current saturation $\left(V_{\text {in }}=V_{\mathrm{s}}\right)$.

Table 1 Measured ion densities $2.0 \mathrm{~cm}$ away from the APP inlet

\begin{tabular}{|c|c|c|}
\hline \multirow{2}{*}{$\begin{array}{c}\text { Fan flow rate } \\
\left(v_{\mathrm{f}} A\right) \\
{\left[10^{2} \mathrm{~cm}^{3} \mathrm{~s}^{-1}\right]}\end{array}$} & \multicolumn{2}{|c|}{$\begin{array}{c}\text { Ion density, } n \\
{\left[10^{6} \mathrm{~cm}^{-3}\right]}\end{array}$} \\
\hline & $\begin{array}{c}\text { Capacitively } \\
\text { coupled }\end{array}$ & $\begin{array}{c}\text { Needle-shaped } \\
\text { electrode }\end{array}$ \\
\hline 1.9 & 4.1 & 16.2 \\
\hline 3.8 & 3.1 & 8.6 \\
\hline 5.6 & 2.8 & 6.7 \\
\hline 7.5 & 2.4 & 5.8 \\
\hline
\end{tabular}
at varying Gerdien fan flow rate.

The $I-V$ characteristics at $7.5 \times 10^{2} \mathrm{~cm}^{-3}$ fan flow is smoothened to obtain its first derivative as shown in Fig. 7. From this, ion mobility can be determined using equation (3).

$$
\mu_{k}=\ln \left(r_{\mathrm{o}} / r_{\mathrm{i}}\right) *(\mathrm{~d} I / \mathrm{d} V) / 2 \pi q_{k} n_{k} l .
$$

Where $l$ is the length of the Gerdien condenser, and $r_{\mathrm{o}}$ and $r_{\mathrm{i}}$ are the outer and inner radii, respectively.

The calculated mobility values are compared from the data derived by Mason et al. [10,11] Major part of the signal is within the range of $\mathrm{O}^{+}, \mathrm{O}_{2}{ }^{+}, \mathrm{Ar}^{+}, \mathrm{N}^{+}$and $\mathrm{N}_{2}{ }^{+}$. The 


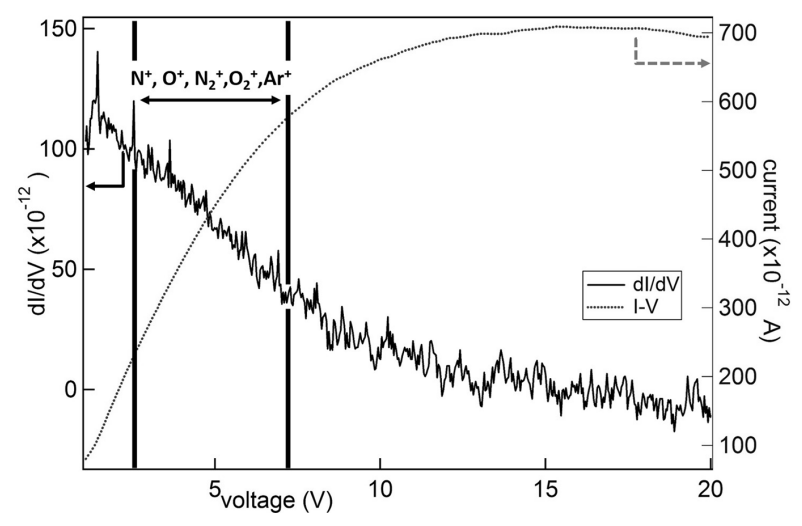

Fig. 7 Ion mobility spectrum derived from $I-V$ characteristics of $7.5 \times 10^{2} \mathrm{~cm}^{-3}$ fan flow rate.

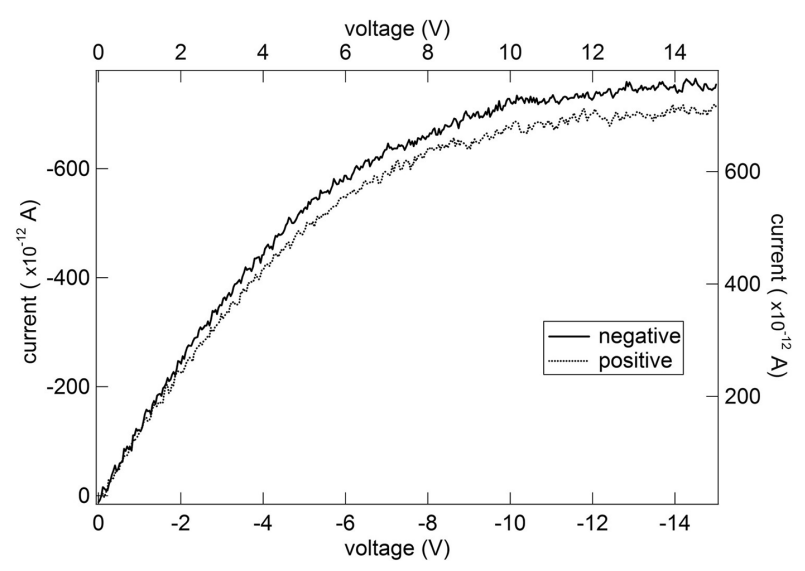

Fig. $8 I-V$ characteristics obtained for negative voltage sweep at $7.5 \times 10^{2} \mathrm{~cm}^{-3}$ fan flow rate.

errors in the determination of the ion mobilities are estimated to be $20 \%$.

\subsection{Negative ion species measurement}

A negative voltage sweep is applied into the outer electrode to obtain information about negative ions present in the plasma. The Gerdien condenser operation is the same even if the electric field is reversed so that negative particles are collected. Through the measured ion mobilities, $\mathrm{O}^{-}$and $\mathrm{O}_{2}{ }^{-}$are assumed to be present in the plasma. However, the result needs further investigation to understand the effect of electron density to the collected saturation current.

\section{Conclusion}

The designed miniaturized Gerdien condenser successfully produced signal to diagnose atmospheric pressure plasmas generated by a $13.56 \mathrm{MHz}$ AC power source. $\mathrm{O}^{-}, \mathrm{O}^{+}, \mathrm{O}_{2}{ }^{-}, \mathrm{O}_{2}{ }^{+}, \mathrm{Ar}^{+}, \mathrm{N}^{+}$and $\mathrm{N}_{2}{ }^{+}$are assumed to be present in the plasma through the analyzed $I-V$ characteristics. The total ion densities of the detected positive ions at pen-to-Gerdien distance of $2.0 \mathrm{~cm}$ for the capacitively coupled configuration ranges from 2 to $4 \times 10^{6} \mathrm{~cm}^{-3}$ and 6 to $16 \times 10^{6} \mathrm{~cm}^{-3}$ for the needle-shaped electrode device. However, the ion density of negative ion species is to be determined by taking the contribution of electron density into account. The results should be reanalyzed based upon precise study of the flow field. It is also of interest to relate the results to Langmuir probe diagnostic technique.

[1] G. Fridman et al., Plasma Processes Polym. 4, 4 (2007).

[2] H.E. Porteanu et al., J. Appl. Phys. 108, 013301 (2010).

[3] C. Fanara et al., J. Phys. D: Appl. Phys. 34, 18 (2001).

[4] M. Cada et al., Surf. Coat. Technol. 174-175, 530 (2003).

[5] A. Pedersen, Tellus XVII 1 (1965).

[6] C. Croskey et al., Proceedings of the 16th ESA Symposium on European Rocket and Balloon Programmes and Related Research, ESA SP-530 (2003).

[7] K. Aplin, Instrumentation for atmospheric ion measurements (PhD Thesis, Department of Meteorology, The University of Reading 2000).

[8] D. Burt, The development of gerdien condenser for sounding rockets in Scientific Report No. 8 (Air Force Cambridge Research Laboratory, Office of Aerospace Research, United States Air Force, May 1967).

[9] J.A. Chalmers, Atmospheric Electricity (2nd ed., Pergamon Press, 1967) p.61-63.

[10] H.W. Ellis et al., At. Data Nucl. Data Tables 17, 177 (1976).

[11] L.A. Viehland et al., At. Data Nucl. Data Tables 60, 37 (1995). 